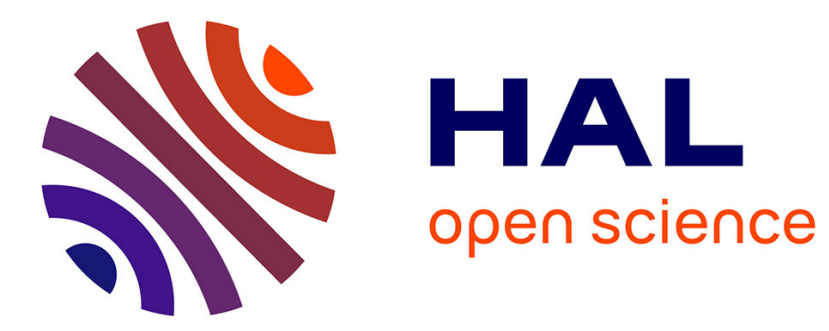

\title{
Sur l'anthropologie économique de Bourdieu et la sociologie de la consommation de Simon Langlois
}

François Gardes

\section{To cite this version:}

François Gardes. Sur l'anthropologie économique de Bourdieu et la sociologie de la consommation de Simon Langlois. 2021. halshs-03325100

\section{HAL Id: halshs-03325100 \\ https://shs.hal.science/halshs-03325100}

Submitted on 24 Aug 2021

HAL is a multi-disciplinary open access archive for the deposit and dissemination of scientific research documents, whether they are published or not. The documents may come from teaching and research institutions in France or abroad, or from public or private research centers.
L'archive ouverte pluridisciplinaire HAL, est destinée au dépôt et à la diffusion de documents scientifiques de niveau recherche, publiés ou non, émanant des établissements d'enseignement et de recherche français ou étrangers, des laboratoires publics ou privés.

\section{(c)(1)}

Distributed under a Creative Commons Attribution| 4.0 International License 


\section{Documents de Travail du

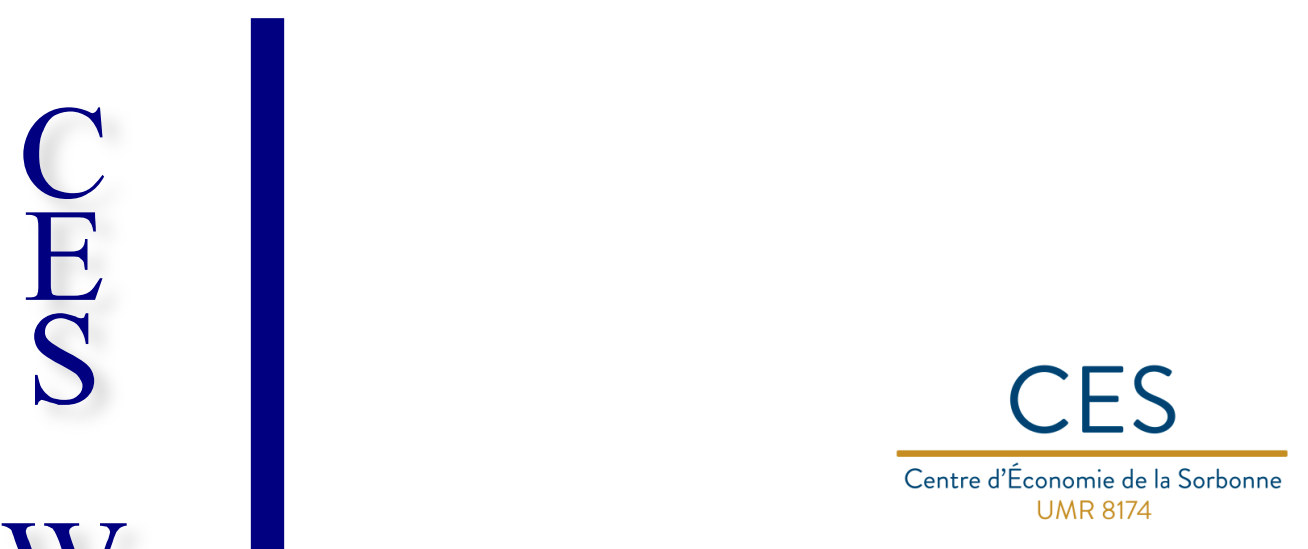

Sur l'anthropologie économique de Bourdieu et la sociologie de la consommation de Simon Langlois

François GARDES

2021.21

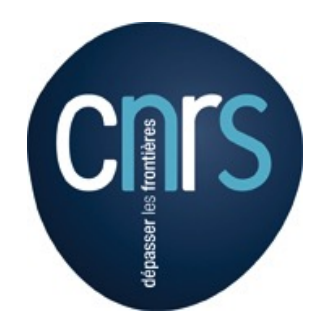




\title{
Sur l'anthropologie économique de Bourdieu et la sociologie de la consommation de Simon Langlois
}

François Gardes

Paris School of Economics, Université Paris 1 Panthéon Sorbonne, Université Catholique de

l'Ouest

Janvier 2021

\begin{abstract}
A la mémoire de Bertrand Lemennicier, grand économiste libéral dont l'œuvre recoupe bien des thèmes de l'anthropologie économique de Bourdieu et des travaux de Langlois.
\end{abstract}

\section{Résumé}

L'examen critique du cours de Pierre Bourdieu sur les fondements sociaux de l'action économique et sa comparaison à la méthodologie suivie par Simon Langlois dans ses analyses des choix de consommation des ménages, engendrent une discussion des méthodologies de test des hypothèses et des conclusions des analyses théoriques dans les diverses sciences sociales et l'exposé succinct de nouveaux modèles qui élargissent la théorie économique des choix individuels, répondant ainsi partiellement aux critiques de Bourdieu.

\section{Introduction}

L'œuvre de Pierre Bourdieu a suscité de nombreuses critiques de la part de sociologues, qui lui reprochent le flou de ses concepts et de ses analyses (habitus, champs de force ou de lutte, capital symbolique), ses travers méthodologiques (déterminisme, attention exclusive à la domination, refus des idéalités au profit des «réalités concrètes »...), sa recherche constante d'une domination intellectuelle et institutionnelle. Elle fait l'objet par ailleurs d'admirations délirantes $^{1}$ (le cours d'anthropologie économique récemment publié est ainsi qualifié par Patrice Bollon, 2018, d'extraordinaire -italiques dans le texte-, d'une importance vitale). La lecture des ouvrages de Bourdieu, et en particulier de ce cours, donne pourtant, au premier abord, l'impression de platitudes hors du commun dans la littérature scientifique, et d'un recollement maladroit de concepts évidents, extraits généralement d'avancées scientifiques modernes (par exemple de concepts de la microéconomie, tel le capital, qui seront exploités en parallèle avec de grossières critiques épistémologiques) ou des œuvres sociologiques classiques (Weber, Mauss et Marx en particulier). La parution de son cours du collège de France de 199293 sur l'anthropologie économique, qu'on peut considérer comme un bilan provisoire de ses

1. «Il essayait de faire comprendre qu'une réalité observable devait être vue comme le produit d'un jeu incessant (mais structuré) entre des forces multiples (quoiqu'ordonnées hiérarchiquement) et composites » (Bollon, 2018) ; « Il a importé dans une discipline naissante et marginale (!) un ensemble assez exceptionnel de ressources » (Marc Joly, 2018). 
analyses dans le domaine des choix économiques, en particulier de consommation, est l'occasion d'une lecture critique de ses analyses de l'homo-oeconomicus qui nous amènera à discuter de quelques principes méthodologiques des sciences économique et sociologique.

Les deux critiques essentielles que Bourdieu fait de l'anthropologie naïve des analyses économiques des choix individuels de consommation portent : (i) sur le fondement irréel »de la rationalité individuelle (ce modèle de rationalité confondant la rationalité du scientifique avec celle de l'ensemble des agents économiques ${ }^{2}$ - critique déjà utilisée par Sartre - et manquant d'encastrement historique dans le passé des individus, qui va constituer leur habitus) et (ii) sur la tentation impérialiste de la science économique, incarnée en particulier pour Bourdieu par Gary Becker et l'Ecole de Chicago. Au-delà de cette thèse centrale qu'on examinera dans les deux premières sections, le cours de Bourdieu fait de nombreuses références à son empirisme assumé, ce qui amène à discuter en troisième section de sa méthodologie et à la comparer aux méthodes empiriques des analyses sociologique et économiques modernes des choix de consommation des ménages. La dernière section discutera des méthodes empiriques de la sociologie et de l'analyse économique de ces choix de consommation et la quatrième d'une proposition de modélisation dépassant les limites de ces analyses.

Selon Bourdieu, il faut opposer à la « formidable arrogance impérialiste » de la science économique (cours, p. 232), non pas des études empiriques particulières permettant d'imposer des correctifs ponctuels, «à la Tycho Bahe », aux lois économiques générales, mais un autre paradigme systématique, une théorie générale de l'action humaine (« fonder et systématiser une théorie anthropologique qui pourrait être opposée à ce paradigme » économique qui « considère comme acquises des choses qui ne le sont pas ») que Bourdieu, dans ses œuvres antérieures et dans ce cours, appliquera principalement aux choix de consommation des ménages : il convient de plus que cette théorie générale de l'action ne soit pas anhistorique, l'homo-oeconomicus étant un «homo-historicus» qui s'ignore (cours du 29 avril 1993³). Les mathématiques qu'utilisent les économistes théoriciens ne font donc que " passer les notions de sens commun, mais maquillées, en catégories scientifiques »: apparait déjà l'insuffisance des modèles statiques, qui ne peuvent rendre compte des changements de goûts et de conditions de choix (exogènes ou provenant des décisions passées des agents) ${ }^{4}$. Mais en même temps, Bourdieu invite à refuser les systèmes fermés, « sorte de défaite de la pensée incapable à organiser le réel sans le réduire à un système préétabli $»^{5}$ et propose «un rejet de la théorie pure, détachée de

2. Cours du 17 juin 1993, page 261.

3. Voir par exemple la réponse à une question des auditeurs sur les invariants chez Lévi-Strauss : «la notion d'invariant est très élastique, elle est très utile pour nommer l'une des intentions de la science sociale qui consiste à chercher, non pas des essences éternelles, mais des invariants transhistoriques. Elle est utile à ce titre mais encore faut-il savoir ce qu'on en fait : elle peut être le masque d'un nouvelle essentialisme anthropologique » - ce qui ne fait guère avancer le smilblic.

4. Ce point sera repris en section 4.

5. Selon Loïc Wacquant, cité par François de Singly dans son article du Magazine Littéraire, 1998. C'est la critique même de la plus grande partie de la «philosophie ancienne » opérée par Bergson, sur laquelle on reviendra en section 3. A la construction d'un système d'ensemble, Bergson propose de substituer une approche particulière (une méthode) dédiée au problème étudié, qui a été sélectionné comme une question essentielle par l'analyste. Il s'agit donc d'abord de découvrir cette question, puis le cheminement qu'il convient de choisir et les faits essentiels qui lui sont reliés (Durkheim parle pour sa part dans un sens proche de « groupes de faits bien circonscrits »), enfin 
tout référent empirique » (L. Wacquant, op. cit.). On analysera plus précisément les problèmes posés par ces méthodes d'analyse, entre théorie et empirie, dans la section 3.

\section{Les thèses centrales du cours d'Anthropologie Economique de Pierre Bourdieu :}

Les quatre premiers cours (d'avril à mai 1993, pages 11 à 115, donc plus d'un tiers de l'ouvrage) sont consacrés à une synthèse de ses analyses sur le don, en discussion des analyses classiques initiés par Mauss et de ses premiers travaux ethnologiques sur l'organisation traditionnelle des échanges en Kabylie. La seule originalité de cette longue analyse concerne sa discussion de la théorie du don proposée par Derrida : la séparation nécessaire du don et du contre-don (pour que le don puisse être vécu comme tel par les deux échangeant) s'opère, selon Bourdieu, par la séparation temporelle entre ces deux actes, ce qui montre l'importance de la dimension temporelle dans l'analyse des échanges : ce ne me semble pas être un apport d'une grande originalité, malgré la présentation enthousiaste que Bourdieu fait de son analyse. Les cinq cours suivants (de mai et juin 1993, pages 115 à 263) reprennent sans beaucoup les élargir l'exposé de ses concepts favoris - champs, distinction, habitus, capital social - avec une discussion assez pauvre de la création des marchés et de leur différenciation, non informée de la littérature économique récente réduite dans son analyse à quelques truismes et la discussion d'une page du manuel d'économie industrielle de Jean Tirole (pour montrer que le grand traité d'économie industrielle ne se soucie pas de définir précisément ce qu'est un marché).

\subsection{Le rôle de l'échange marchand et la dimension symbolique des biens}

La critique de l'économique de Bourdieu commence donc par celle de la modélisation de l'échange, entendu seulement, selon lui, comme un échange marchand, ce qui est contredit par l'existence d'échanges par don dans les sociétés précapitalistes et dans la sphère familiale, évoquée dans la deuxième leçon : la famille est un ilot de $\varphi \imath \lambda ı \alpha$ dans un univers de calcul, le principal lieu de refuge des échanges non marchands ${ }^{6}$. Le don révèle la dimension symbolique des biens et services économiques, qui deviendra l'un des concepts abondamment utilisé par Bourdieu. Cette dimension symbolique, bien connue des économistes ${ }^{7}$ depuis Veblen (auteur discuté trop succinctement par Bourdieu), consiste en l'occurrence en l'intégration de la relation

\footnotetext{
de n'exposer les résultats obtenus qu'une fois une pleine compréhension de la question obtenue : « on n'est jamais tenu de faire un livre », dernière phrase de son dernier ouvrage.

6. L'inverse est proposé par Luc Ferry : l'invention moderne de l'amour conjugal (d'abord au sein de la bourgeoisie du fait du desserrement de la contrainte financière) s'accompagne naturellement d'une extension des échanges d'amitié.

7. Ce que semble ignorer Bourdieu, voir la page 228 du cours par exemple : « la théorie économique pure et dure qui entend réduire le symbolique à l'économique a quelque chose de comique dans une société où le symbolique est au fondement même des mécanismes économiques les plus fondamentaux », ce que n'ont ignoré ni les microéconomistes modernes, ni les théoriciens et les praticiens du marketing. Bourdieu cite par exemple (cours du 29 avril 1993, page 73) un article d'économistes sur le don qu'il « avait l'intention d'évoquer parce qu'il l'a énervé » mais n'explicite pas sa critique : «Je vous en donne simplement la référence, en espérant qu'il vous énervera ». Rires dans la salle, indique l'éditeur. Les groupies sont tous présents.
} 
interpersonnelle établie par le don, avec un contre-don qui, sans être obligatoire, est supposé se produire dans l'avenir ${ }^{8}$. Bourdieu semble finalement proposer implicitement par endroits l'idée d'un retour aux relations de don dans les sociétés modernes, sans discuter aucunement le fait que les structures interpersonnelles de don y seraient terriblement complexes, du fait de la diversité des échanges, et donc ingérables par les agents sans l'utilisation d'une monnaie d'échange commune. Toute cette analyse, non sans intérêt, pêche néanmoins par une discussion très schématique de l'ensemble des problèmes posés par la coexistence d'échanges par don ou sur un marché : l'important pour Bourdieu semble être d'introduire comme caractéristique essentielle de tout bien échangé sa nature symbolique, qu'il sera facile d'invoquer à tout bout de champ dès qu'un problème de compréhension des échanges marchands se pose (correspondant le plus souvent à une difficulté apparente : ce type de problèmes est lié souvent à des contraintes non perçues ou des coûts non monétaires, deux points que l'on évoquera en dernière section).

Il s'agit donc, pour corriger cet aveuglement de l'analyse économique, d'abord de construire une «théorie de l'économie des biens symboliques comme ordre économique ayant sa logique propre » (incluant le capital symbolique, les profits symboliques, les intentions symboliques - dispositions généreuses), secondement de réintroduire le temps (que Bourdieu dénommera souvent «l'histoire ») dans l'analyse (ce que fait selon lui la succession des deux temps du don et du contre-don), enfin de considérer une autre philosophie de l'agent et de l'action (souvent non consciente ni d'intention, mais soumise à des dispositions et à un habitus hérité des déterminations passées, en particulier familiales).

Généralisant l'observation (théorisée) que le don charge les biens d'un symbole social', il fait l'hypothèse que tout échange (par don ou peut-être sur un marché) agit de même, l'objet échangé acquérant, au fil des échanges, une charge croissante de caractéristiques symboliques : ceci est évidemment plus une hypothèse qu'une conséquence nécessaire, les dons (ou échanges) successifs pouvant créer des charges symboliques qui se remplacent, se renforcent ou s'annulent. Or ceci est une thèse centrale de Bourdieu : tout échange économique modifie la nature symbolique du bien (le fait que ce point importe à Bourdieu s'observe en particulier par la place, presque de moitié, qu'il donne à l'analyse du don dans son cours). Cette analyse est en fait très imprécise : il reste par exemple à savoir si ces changements sont également perçus par tous les acteurs sociaux et s'ils valorisent également ces charges symboliques. Si c'est le cas, le bien pourrait être affecté d'un prix virtuel (positif ou négatif) qui s'ajouterait à son prix monétaire (supposé uniforme dans la société), prix virtuel qu'on pourrait éventuellement révéler en comparant les demandes et offres avant et après charge symbolique. Dans la mesure où les charges symboliques opérées par les échanges ne sont pas perçues et valorisées identiquement

8. Il est curieux que Bourdieu, auteur par ailleurs d'un ouvrage sur Heidegger, et citant assez fréquemment Sartre (mais pas pour La critique de la Raison Dialectique), ne propose pas une lecture existentialiste du don : l'idée par exemple que le don produit l'altérité de l'autre, c'est-à-dire concourt à la création par l'individu de son environnement social, le disposant par rapport à un monde au sens d'Heidegger (au lieu de sa situation originelle face à un néant désespérant), ou à l'autre (au sens de Sartre) ainsi maitrisé par l'obligation du contre-don - à la différence de l'échange marchand, qui rend immédiatement ce que l'on projette.

9. «Le don (comme une magie), transforme les choses échangées, qui se mettent à avoir une valeur sacrée d'autant plus sacrée qu'elle circule davantage. Il transforme les gens qui échangent, la perception qu'on a d'eux... En un sens il change tout. » (cours du 6 mai 1993, p. 112). Dans la leçon suivante, Bourdieu affirmera même (en négligeant d'y apporter une preuve précise) que «les échanges symboliques sont l'instrument unique, quasiexclusif, de construction de relations sociales durables ». 
par les acteurs sociaux, ces prix virtuels seraient individualisés et agiraient (en terme d'analyse économique) comme les prix virtuels qu'on peut attacher aux contrainte subies par l'agent (par exemple de rationnement, qui correspond à une hausse du prix accepté par le demandeur si le bien était dé-rationné) ou à ses ressources non-monétaires (d'information, de temps libre...). On trouvera un développement de cette analyse en section 4.

\subsection{Le temps de l'histoire}

La succession des actions de l'agent dans le champ social va donc modifier, et la nature des biens, et les relations sociales qu'il entretient avec les autres agents. Il est donc absurde de chercher un équilibre économique en demeurant au niveau statique d'un ensemble de biens, de préférences et de ressources individuelles : l'analyse complète de ces actions doit se faire en explicitant leur insertion temporelle dans une histoire jamais achevée ${ }^{10}$. On ne trouve pas de référence dans le cours à un mode de construction générale de ce type d'analyse dynamique, Bourdieu intégrant semble-t-il toute cette dynamique dans la constitution et les évolutions de l'habitus des agents et des champs dans lesquels ils s'insèrent - deux concepts dont il parle comme d'avancées majeures de l'analyse des actions sociales ${ }^{11}$.

\subsection{L'habitus et les champs sociaux}

La référence répétée, dans le cours et toute l'œuvre, à l'habitus (défini, d'une manière qu'on peut juger trop imprécise, comme un «système de préférences socialement constitué » ou comme « ce que l'on a acquis, mais qui s'est incarné de façon durable sous forme de disposition permanente » - système de dispositions accordé à un jeu dans les institutions) qui se (ou que se ?) constitue (chaque agent) dans le déroulement de sa vie sociale (avec un poids majeur de ses expériences familiales initiales - affirmation dont je n'ai pas trouvé de justification théorique ou empirique dans le cours), ne permet pas facilement de fixer le niveau d'action de cet habitus : doit-on le situer comme une détermination profonde des préférences qui se révèlent dans les choix de l'agent, ou simplement dans ses comportements, tels qu'ils apparaissent au fil de ses expériences dans des champs sociaux successif ? Comment le relier aux analyses psychologiques ou économiques (de Stigler et Becker, 1977, par exemple) de formation des préférences (engendrant des préférences endogènes aux choix passés et aux situations vécues) : Bourdieu, qui s'oppose clairement à l'impérialisme supposé des méthodes d'analyse de Becker, n'aborde pas frontalement cette confrontation, semblant considérer que la généralité de son concept d'habitus ne doit pas s'évaporer dans des représentations trop particulières ou dans une comparaison à des analyses trop formelles. Par ailleurs, ni le degré de stabilité de cet habitus ni

10. Bourdieu ne fait aucune référence sur ce point, qu'il laisse très imprécis, aux analyses très développées dédiées par Sartre (Critique de la Raison Dialectique) à l'homme se faisant en société. Il se réfère par contre dès la première leçon (p. 42) à la critique de la science sociale comme "monothétique et non polythétique », la théorie sociale consistant généralement à instantanéiser, synchoniser et détruire le temps de l'action, « ce qui explique le décalage entre le modèle et l'expérience ». Ce type de science sociale pêche à «placer dans les agents des pensées de savant » (comme l'homo-oeconomicus), ce dont s'exonère semble-t-il Bourdieu lui-même.

11. Mais qui me semble à dire vrai bien banales dans leur généralité, dénominations nouvelles de concepts habituels aux sciences du comportement et des choix en société. 
les règles de son évolution ne sont précisées (sauf peut-être dans des études spécialisées antérieures).

L'habitus amène l'individu à des dispositions qu'il appliquera dans le champ correspondant à sa décision. Le concept de champ semble, du moins à la lecture du Cours, tout aussi imprécis, malgré le vocabulaire censé le caractériser («l'architecture des champs », jamais précisée) : tout domaine ou environnement de choix semble pouvoir être considéré comme un champ. Bourdieu explique longuement l'avoir préféré au concept de marché, qui se réduit à un échange de biens de valeur égale ${ }^{12}$, dissociant ainsi l'acte de communication de l'acte de pouvoir présents dans l'échange, l'unique propriété du marché étant (selon Bourdieu) de fixer la valeur des choses (voir les pages 167-8). Bourdieu n'évoque donc pas la vertu d'équilibrage des offres et demandes sur le marché et le rôle des prix dans le passage d'un équilibre à l'autre ${ }^{13}$. Sa défense du mot choisi est assez singulière :

Weber entend le marché d'une manière très particulière ${ }^{14}$, mais il garde le mot. C'est un problème dans la recherche : si l'on garde un mot en lui donnant un sens différent du sens ordinaire, les imbéciles vont le rabattre sur le sens ordinaire. Weber a pris ce risque. J'ai pour ma part hésité très longtemps entre «champ » et «marché », parce-que conserver le mot marché a évidemment des avantages (cela dispense de devoir tout raconter), mais, au total, les coûts sont largement supérieurs aux profit. Ce n'est pas dans un souci de distinction que je dis « habitus » plutôt qu' « habitude», « champ » plutôt que «marché », mais pour éviter les retours en force de la philosophie immanente à un mot. (Bourdieu, 2017, p. 209-210)

Il n'y a donc pas de différence essentielle entre champ et marché (tout marché est un champ à la Bourdieu, et les économistes n'ont jamais douté que les marchés étaient des champs de lutte et de pouvoirs), toute la différence tenant à la nature conflictuelle, historique et multidimensionnelle (par l'incorporation de caractéristiques symboliques dans les biens échangés) de toute relation entre les partenaires d'un échange marchand ${ }^{15}$. Le champ de Bourdieu se caractérise ainsi (voir les pages 177 à 194) par la présence (essentielle) de barrières à l'entrée ( «Dans tout champ, il est question des limites du champ : cette phrase n'a l'air de rien mais, d'un point de vue empirique, elle est extrêmement importante », p. 185), où les actions des acteurs sont toujours distinctives (pour que l'agent existe par sa différence) et qu'on ne peut définir définitivement : «Un champ, s'il est le lieu de luttes entre des populations, n'est pas une population. Un effet des champs est précisément de dire qui en fait ou non partie à un certain moment et sous certaines conditions. Dans un champ, il est question à chaque moment

12. Notons ici une imprécision dramatique de la notion d'égalité des valeurs échangées, qui était la conception de l'échange marchand de la scholastique dont la révolution nominaliste, à partir de Duns Scot (au $13^{\text {ième }}$ siècle), va montrer l'insuffisance en introduisant l'idée que les gains des agents échangeant ne sont pas nécessairement égaux (voir par exemple Karl Pribam, 1998, Chapitre 3).

13. La notion d'équilibre apparait justement dans les analyses du commerce international après qu'ait disparu le modèle scholastique de l'économie (Pribam, 1998).

14. En distinguant deux moments : la concurrence entre les producteurs et, en deuxième phase, l'échange avec le partenaire.

15. Notons cependant qu'en rejetant l'identification (même partielle) des champs à des marchés, Bourdieu s'interdit de penser toute dynamique de ré-équilibrage par des coûts ou des prix généralisés (monétaires ou virtuelles), et donc l'aspect dual, dans une dimension de prix, de ces champs sociaux. 
des limites du champ ». Indépendamment de la lourdeur de l'expression (permanente dans ce cours), le concept ne se précise guère au fur et à mesure des explicitations, d'autant plus que les champs particuliers qui sont décrits dans le cours ne se distinguent pas nettement, pour le profane, de ce qu'on pourrait appeler marchés ou domaines sociaux. Il semble que ce concept de champ soit considéré comme l'évidence même par Bourdieu, d'un point de vue introspectif ou selon les analyses empiriques de toute une vie, et qu'il ne trouve que des inconvénients à tenter de le réduire à quelques caractéristiques ou propriétés précises ${ }^{16}$. «Une définition opératoire serait, comme disait Spinoza, «l'asile de l'ignorance». Dire : "j'appellerai intellectuels » puis énumérer (ceux que j'appellerai ainsi), c'est catastrophique parce qu'on détruit l'objet avant même de l'avoir étudié. C'est ce que fait Tirole : il choisit d'appeler « marché » ce qu'il juge bon d'appeler marché pour les usage qu'il a à en faire à un moment donné ${ }^{17} \gg$. Mais comment étudier cet objet si on ne le définit pas préalablement ? En le créant, selon Bourdieu, « conformément à ses structures », par une sorte de « constructivisme réaliste selon lequel la science construit, mais conformément aux structures objectives de l'objet »qu'on atteint comment?

Là se trouve sans doute l'une des clés pour comprendre la démarche scientifique de Bourdieu : il convient d'avoir une intuition préalable de ces «structures objectives de l'objet d'étude », et cette vue d'ensemble intuitive doit dépasser en dignité ce que suggèrerait une observation empirique, a-théorique, à partir d'une délimitation plausible et a priori de l'objet d'étude (par exemple les populations violentes et asociales, qu'on peut semble-t-il repérer assez aisément). Un de ses premiers collaborateurs, universellement considéré comme un sociologue de grande qualité, disait avec force avoir vécu le moment même où Bourdieu afficha que ses idées a priori surpassaient la réalité : alors qu'on lui objectait pendant un séminaire un fait empirique contraire à son discours, Bourdieu s'était écrié : «ce qui est dans la tête de Pierre Bourdieu est plus important qu'une réalité empirique» (considérée sans doute comme partielle) ${ }^{18}$. Indépendamment de la superbe de la réponse (dont on peut excuser le caractère gascon), elle traduit nous semble-t-il un postulat essentiel de sa méthode d'investigation, qui n'utilise souvent les faits empiriques que pour conforter un jugement déjà bien établi - mais dont on n'est pas obligé d'accepter l'argument d'autorité, surtout venant de son fabricant.

Habitus et champ, comme les «dispositions » individuelles qui s'en déduisent, sont finalement plus des mots que des concepts nouveaux et précis : manière de parler savante pour énoncer beaucoup d'évidences ${ }^{19}$. Bourdieu s'en excuse par endroit : « ce que j’ai essayé de

16. «J'hésite toujours à donner une définition rigoureuse qui risque de paraître inintelligible et inutilement compliquée », p. 179 ; «quand il s'agit d'un champ, donner une définition, une délimitation, c'est considérer comme résolu le problème même que pose l'existence d'un champ », p. 185.

17. Critique absolument ridicule pour quiconque a lu les travaux de Tirole et Laffont.

18. Péguy, De la Grippe : «Vous n'avez pas pensé que c'étaient les évènements qui avaient tort et l'hypothèse qui avait quand même raison? ».

19. Par exemple en page $213:$ : Sur le marché de la coiffure, il y a une structure de l'espace de production, avec, à un bout, Carita et, à l'autre bout, le coiffeur de quartier ; en face, il y a une structure de l'espace de consommation et les goûts, comme les offres, sont dispersées » : l'analyse s'arrête là (elle pouvait par exemple se continuer par une discussion de l'oligopole d'Hotelling), ayant sans doute atteint le point ultime du saisissement sociologique ; ou, en page 206, ce commentaire sur Max Weber : «quand il dit «désir » ou «concurrence pour des choses désirées par d'autres », il entend désir socialement constitué dans la référence à un champ, c'est-à-dire ce que je 
faire, c'est de donner une allure plus systématique aux lectures que j'ai pu faire $»^{20}$. Quel intérêt à cet habillage ?

\section{La critique bourdieusienne de l'analyse économique :}

\subsection{Rationalité supposée de l'agent calculateur et informé}

Bourdieu aime à dénommer les «RATS » les agents économiques de la Rational Action Theory, qui, selon lui, les suppose généralement (à l'exception d'Herbert Simon ${ }^{21}$ ) capables des mêmes calculs que les modèles les plus sophistiqués (et l'économiste lui-même), parfaitement informés et sans insertion sociale particulière ni réseau d'interactions sociales. Cette description est mal informée des analyses économiques menées depuis presque un siècle par les économistes du courant néo-classique ou les post-keynésiens, par exemple sur les choix en incertitude (Knight, 1938), la recherche d'information (Stigler, 1960), les interactions sociales (Becker, 1974), la rationalité statistique des choix individuels (Becker, 1962) et les tests empiriques de la rationalité des choix individuels, les procédures de choix non optimisatrices (correspondant à des préférences non intégrables, c'est-à-dire non issues de la maximisation d'une utilité : théorie des préférences révélées de Samuelson, 1938), les procédures collectives des choix effectués par des groupes sociaux, tels les ménages (modèle collectif des choix familiaux, Becker, 1981 et Browning et al., 2014), les analyses empiriques des processus d'anticipation des ménages. L'homo-oeconomicus se trouve donc réduit à une monade calculatrice, sans porte ni fenêtre, dont on ne sait comment il réussit néanmoins à établir quelques liens sociaux durables (la seule interaction découlant du don, ou d'une autre rapport marchand, qui établit un «lien enchanté tissé dans la durée » ${ }^{22,23}$ ). Par ailleurs, toute analyse économique dans la tradition néo-classique s'accompagne effectivement d'une hypothèse de séparation des caractéristiques proprement économiques (liées à la rareté) des biens échangés et de la nature de l'échange, tout comme le physicien étudiant la plasticité d'un matériau la supposera, dans un premier temps, indépendante de ses propriétés électriques. Certes, cette hypothèse de séparabilité est souvent implicite, mais elle ne perturbe généralement pas le calcul technique basé sur les raretés (si c'est le cas, le modélisateur sera amené naturellement à revenir sur cette séparabilité). La critique de Bourdieu perd ainsi beaucoup de son intérêt et peut être rangée auprès des jugements journalistiques sur l'irrationalité des acteurs de l'économie.

Rien n'est dit non plus dans le cours sur le rôle des prix dans la transmission d'information à ces acteurs, qui auraient pu par exemple commencer par une discussion des

mets sous l'expression de «libido sociale» (libido dominandi, libido sciendi, libido auri, etc.). Je rappelle au passage que ce que j'entends par « intérêt », c'est une libido socialement constituée, c'est-à-dire une disposition à aimer des choses socialement constituées comme aimables dans un état donné d'un univers social déterminé : l'or dans le cas du champ économique, etc. ». Je n'opère ces longues citations que pour indiquer l'extrême généralité et l'imprécision de la plus grande partie des analyses particulières présentées dans ce cours.

20. Il n'est pas toujours aussi modeste : «la petite révolution que représente la pensée en termes de champs » (p. 196).

21. Bourdieu ne cite jamais Raymond Boudon dans ce cours.

22. Les descriptions de Bourdieu ont souvent ce parfum de mystère et d'imprécision, qui gêne la compréhension. 23. Le don s'accompagnant de l'obligation d'être obligeant à l'avenir fournit cet enchantement qui diffère de la brutalité de l'échange marchand. Rien n'est dit d'une relation qui mélangerait don et rapport marchand. 
analyses d'Hayek sur l'importance des marchés dans cette transmission des informations sur la rareté des biens. L'habitus et les dispositions qui s'ensuivent dans sa relation avec un champ semblent suffire à comprendre les modes de choix des acteurs, comme si la recherche d'information ne pouvait être séparée de ces tendances profondes de l'individu. C'est un peu le sens des critiques très fines faites par Jeffrey Alexander (1995) d'une théorie trop générale, trop ambitieuse et jamais totalement appliquée, au lieu de construire une théorie analytiquement plus complexe des modes de décisions assortie d'un mode de preuve, statistique ou non, clairement affiché. La conséquence, selon Alexander (voir la page 24 de son ouvrage), de cet effort inabouti est la réintroduction du comportementalisme (par une stratégisation de l'action), l'assujettissement du sujet à des codes symboliques surplombants (réintroduisant le structuralisme) et sa soumission à une base matérielle sous-jacente (réintroduisant le marxisme orthodoxe ${ }^{24}$ ). Les champs sociaux de Bourdieu, tels qu'il les décrit dans ses travaux empiriques, sont finalement largement assujettis à des structures économiques et finalement peu autonomes par rapport aux domaines ou marchés qu'on analyse en économie, donc sans intérêt théorique ni empirique.

\subsection{Insuffisance de l'analyse économique des marchés et de la théorie économique des contrats}

Les analyses économiques des marchés et des contrats que discute Bourdieu dans ses cours des 3 et 10 Juin (après un rappel des analyses du marché de Max Weber) concernent, lorsqu'elles sont plus que des jugements à l'emporte-pièce sur l'économie industrielle moderne, les travaux de Chamberlin et Joan Robinson, datant des années 30. Rien n'est dit des premières études d'économie industrielle de Chicago (par Stigler) et du développement tant théorique qu'appliqué de ce domaine depuis 1970. La théorie économique moderne des contrats est à peine citée. Ce qui est déjà peu admissible dans un article de grande diffusion l'est encore moins dans un cours censé produire un jugement informé sur le rôle des marchés aujourd'hui et leur éventuelle nocivité.

Selon Bourdieu, l'analyse économique des marchés pêche d'abord par l'absence de définition précise de ce qu'est un marché du point de vue de cette analyse. Il repère en particulier cette absence, aux pages 171-172, dans le manuel d'économie industrielle de Jean Tirole qui considère que les limites du marché, en termes de biens concernés, sont malaisément définies par l'importance des élasticités-prix croisées entre ces biens, dont l'estimation est généralement peu robuste. Il faudra donc postuler a priori l'existence d'un marché et le circonscrire, cette existence étant nécessaire à toute analyse d'équilibre partiel : un marché sera le lieu, éventuellement dématérialisé, sur lequel peuvent s'effectuer des échanges entre agents pour un ensemble de biens qui apparaitra dans ces échanges : il y a marché dès qu'il existe une possibilité d'échange, la manière dont les échanges s'effectuent définissant les propriétés de ce

24. «Dans ses études ethnographiques postérieures, comme dans ses analyses sur la société kabyle, Bourdieu exhibe la même incapacité à conceptualiser une distance, un espace critique, entre les structures mentales et les conditions sociales dont elles émergent: «des conditions d'existence différentes produisent des habitus différents » (Bourdieu, La Distinction, p. 190). La pensée n'est rien de plus que le reflet inversé de la vie » (Alexander, p. 24). 
marché. Notons que cette nécessité, selon Bourdieu, de définir précisément le marché sur lequel portera l'analyse est quelque peu contradictoire avec la recommandation fréquente de Bourdieu, de ne point enfermer l'objet d'étude dans une définition préalable, ce qui en restreint donc la portée. En deuxième lieu, l'analyse purement économique de l'échange marchand de deux biens de valeur (marchande) égale dissocie l'acte de communication de l'acte de pouvoir, sa fonction essentielle étant de fixer la valeur des choses. Toute la dynamique des échanges marchands se trouve donc négligée dans cette conception étroite du marché fixant la valeur économique des choses. On remarquera que cette « valeur » n'est que temporaire, conditionnelle au mécanisme ponctuel d'équilibrage des offres et demandes par les prix. Bourdieu ne parle jamais, dans son analyse de l'antagonisme entre les relations sociales traditionnelles (sans procédure précise de calcul par les acteurs) et les échanges économiques caractérisés par un calcul précis des valeurs, de la complexité des rapports marchands (indépendamment de leur dimension sociale), qui s'insèrent dans un système d'échange général et dans une dynamique où les échanges passés influencent les présents (par des contrats explicites ou implicites entre les échangeurs) autant que les anticipations des évolutions futures des prix et de la nature des biens. Or, cette « logique économique » qui fait l'objet de sa critique n'existe que du fait de l'existence de prix marchands, dont les équilibrages statique et dynamique dépendent des formes de marché. L'absence d'analyse du rôle des prix monétaires (et en particulier du fait qu'ils peuvent intégrer la valeur symbolique des biens) constitue le manque essentiel de cette critique par Bourdieu de l'analyse économique des marchés.

Quant à la théorie économique moderne des contrats (évoquée dans la cinquième leçon), elle ne peut en aucun cas selon Bourdieu (mais ceci est une assertion non prouvée par une quelconque analyse de la littérature) suffire à expliquer la construction d'une société durable.

\subsection{Les faiblesses méthodologiques de la science économique moderne selon Bourdieu : une multitude de modèles partiels fabriqués par des « petits modélisateurs »}

A l'issue du cours, Bourdieu conclut que les travaux économiques récents n'ont pas d'horizon : ils s'étendent par l'ajout de modèles limités et indépendants, sans réelle prise sur une réalité complexe, que construisent des techniciens dont le langage mathématique (Bourdieu ne discute pas du mode de preuve de ces modèles) n'est que l'habillage d'une analyse incapable de repenser ses fondements implicites: agent calculateur, échange marchand généralisé, insertion sociale des acteurs négligée, temps absent. L'analyse économique n'est donc pas une science dans la mesure où, si elle a développé des techniques spécialisées de recherche des faits et d'inférence causale (dégagement de faits vérifiables par l'observation ou l'expérimentation auxquels sont appliquées des méthodes acceptables d'inférence logique que peuvent opérer des spécialistes formés à ces méthodes), elle manque la dimension sociale essentielle de l'activité et réduit l'acteur à une machine calculatrice. Elle est aujourd'hui le champs d'activité de « petits économistes appliqués », bricoleurs de modèles fondés sur la logique d'un choix économique indépendant des autres insertions sociales de l'agent (que les pédants nommeront « incrustation sociale $\gg)$.

Les travaux de Gary Becker, qui recoupent largement des questions habituellement soumises à des études sociologiques, ne répondent pas, selon Bourdieu, à sa demande de fonder 
une nouvelle analyse des choix économiques qui dépasse les présupposés de l'analyse économique académique (économistes du main stream et hétérodoxes étant mis sur le même plan : ces derniers se contentent d'apporter des correctifs à la Tycho Brahe, cherchant à sauver un paradigme qui est intrinsèquement ruiné par ses fondements implicites). Outre que le projet d'extension des modèles économiques à des phénomènes sociologiques dénote une insupportable volonté impérialiste (critiquable sans doute du fait que la dimension économique de ces phénomènes n'est pas l'essentiel), le fait de considérer que des choix individuels tels que l'action criminelle, le choix d'un conjoint ou toute forme d'interaction sociale peuvent être analysés comme des interactions d'offre et demande sur un marché, avec une régulation par des prix, est profondément faux par rapport aux processus réels de la décision individuelle. La critique de Bourdieu tient en fait à son refus du «mythe de l'impérialisme du marché » (voir page 125) : il s'agit là, non pas d'un simple refus d'application d'un modèle de marché à un comportement a priori non économique, mais de l'hypothèse, qu'il attribue autant à Marx qu'à Simmel, que l'équivalence monétaire opérée par la mise sur le marché d'un bien économique détruit les valeurs non économiques de ce bien : l'intérêt, selon Marx, envahit tout et ronge peu à peu toutes les relations sociales (il n'y a donc pas d'autre moyen que de l'interdire), l'équivalence monétaire impose l'idée que tout est marchandise et donc achetable ${ }^{25}$, « thèse qui va de soi chez les théoriciens néo-classiques » bien qu'elle soit totalement démentie par les faits. Pourtant, Bourdieu a su importer de l'analyse économique tous les concepts (dont l'investissement ou le capital social) sur lesquels il a fondé ses analyses, et le fait qu'un modèle de marché permette de comprendre certains des mécanismes sociaux à l'œuvre dans ces décisions lui confère un statut d'explication «comme si » (as if) conforme à l'ambition modeste - des analyses économiques (selon le critère méthodologique proposé par Milton Friedman $)^{26}$.

\section{Comparaison aux principes et méthodes des analyses sociologiques de la consommation des ménages menées par Simon Langlois :}

\subsection{Une méthode bergsonnienne ${ }^{27}$ :}

On ne trouve pas, dans les nombreux travaux de Simon Langlois consacrés aux choix de consommation des ménages (articles scientifiques ou courts articles de son blog récent de l'Université Laval, 2019), de recherche systématique d'un système fermé complet qui s'adapterait (au prix d'une généralité qui réduit considérablement les explications de Bourdieu) aux divers problèmes et aux périodes étudiés. La méthode d'analyse s'adapte au contraire aux conditions du problème étudié (méthodologie certainement issue de sa formation puis de sa collaboration avec Raymond Boudon) : les méthodes statistiques utilisées vont ainsi de l'établissement de typologies (évoluant au cours des quarante-cinq années de sa recherche), à des analyses démographiques des types ainsi constitués (permettant de mettre à jour les effets de structure à l'œuvre en longue période), à l'interprétation de modèles de consommation

25. Amenant par exemple à justifier la prostitution ou provoquant la corruption du milieu politique.

26. Il semble par moment que Bourdieu, considérant (cours du $1^{\text {ier }}$ avril) que «ce que nous pensons est lié aux positions que nous occupons dans l'espace social», applique cette réserve aux économistes, engagés dans les conseils aux princes ou à l'industrie, et dans ce cas, on ne peut en attendre une analyse objective et communicable à tous. Outre le fait que la plupart des économistes académiques ne sont pas dans cette situation, la même réserve vaut en fait pour toutes les sciences sociales.

27. Voir l'analyse proposée dans la section 4.2. 
estimés (voir par exemple dans l'ouvrage précité la discussion des déterminants du bien-être exprimé), à l'analyse des réseaux (son article de 1977 préfigure la littérature abondante sur ce sujet, en particulier en économie, de la dernière trentaine d'années).

L'article publié au début de sa carrière (en 1977, sur l'utilisation de réseaux personnels de relations sociales pour obtenir des informations sur les offres d'emploi) fait la preuve d'une analyse très précautionneuse, avec une description précise de la nature du sous échantillon de l'enquête publique qui a été utilisée. Il est également frappant que des notions qui ont été développées ultérieurement dans les analyses formelles des réseaux (formalisée par la théorie des graphes), tels la longueur des chaines d'information et l'influence de la position des individus dans le réseau, y soient utilisées.

Une seconde caractéristique bergsonienne de son œuvre tient à son constant souci d'étendre les possibilités d'observation par la construction de bases de données originales, dont le modèle est donné par son pseudo-panel, étendu sur plus de cinquante années (depuis 1969), des enquêtes de consommation canadiennes. Ce type de matériau statistique permet l'étude, à un niveau relativement désagrégé des biens et des consommateurs, des évolutions de longue période des comportements de consommation, et donc d'apporter une réponse empirique à des questions généralement laissées au niveau d'hypothèse, telle celle de la convergence des comportements de consommation. Il reste à souhaiter que cette statistique exceptionnelle soit prolongée et que l'exemple de ce type de données soit étendu à d'autres enquêtes ${ }^{28}$.

\subsection{Le disciple de Boudon et de Tocqueville}

La construction de sa thèse à Paris sous la direction de Raymond Boudon a fermement ancré Langlois dans un paradigme de rationalité extensive des choix des acteurs sociaux et de diversité des cultures qui lui a permis d'étudier avec rigueur des problèmes très divers liés aux comportements individuels tout en conservant un cadre d'analyse commun à ces travaux. C'est en effet l'un des inconvénients des analyses critiques de la rationalité et plus généralement du cadre de l'analyse économique des choix en situation de rareté, que d'interdire les comparaisons entre des études trop liées à des choix méthodologiques ad hoc (décidés en fonction de la critique qu'on souhaite apporter à la théorie dominante). La confiance dont Langlois témoigne dans toute son œuvre en la pertinence du modèle de rationalité de Boudon, précis mais assez flexible pour s'adapter à des choix aussi différents que ceux de l'éducation ou de l'usage du revenu familial, unifie ainsi son œuvre. Parallèlement, une approche à la fois modeste et obstinée, se fondant sur toutes les dimensions des évolutions sociétales complexes, a permis à Tocqueville de révéler les fondements historiques de ces mouvements profonds. Les travaux de Langlois - ceci apparait par exemple clairement dans les courts articles de l'ouvrage précité révèlent cette même attention au réel caché derrière les phénomènes, qu'ils soient

28. Un exemple montrera le type de difficultés que le chercheur rencontre lorsqu'il souhaite analyser des évolutions longues à un niveau désagrégé : des enquêtes de consommation détaillées ont été effectuées annuellement (sur plusieurs dizaines de ménages) en Pologne depuis la dernière guerre mondiale (elles avaient une grande importance dans la planification centralisée). Il serait donc possible d'utiliser ces données d'enquête, par exemple pour examiner l'effet des rationnements, mais les enquêtes étaient supprimées des supports informatiques (installés sur des rubans électroniques à l'époque) lorsque ces supports se trouvaient saturés, ce qui a fait disparaître trente ans de statistiques originales (partiellement panélisées sur quatre années). Les fonctionnaires des organismes internationaux qui visitaient la Pologne à l'époque n'ont su fournir (par ignorance du problème ou peut-être refus des autorités) le matériel informatique qui aurait permis d'éviter ces destructions. 
statistiquement constatés ou issus d'une perception immédiate plus intuitive. La grande diversité des sujets abordés par Langlois trouve ainsi, dans ces deux influences assumées, une grande unité. Elles lui permettent également de rester dans l'assise des analyses des pères fondateurs (Durkheim, Mauss, Halbwachs qu'il cite fréquemment) sans chercher à s'en distinguer artificiellement, mais plutôt en retrouvant dans ses propres recherches certaines de leurs intuitions.

L'analyse des déterminants du vote pour l'indépendance du Québec proposée par Langlois (dans son ouvrages Les Raisons Fortes) constitue un exemple typique de cette méthodologie durkheimienne basée sur une typologie des agents : l'étude des caractéristiques majoritaires des électeurs en termes d'âge, de langue maternelle, d'activité ou de statut d'immigrant permet de saisir les raisons essentielles des votes. On suppose donc, dans la tradition de Durkheim, que ces caractéristiques sont liées à des raisons profondes, par exemple de craindre les conséquences économiques (perte de la rente fédérale, etc.) de l'indépendance quand on est âgé, en faible revenu ou immigré récent. La structure des données repérées par l'enquête sert à révéler ces motivations profondes (qui pourront être ultérieurement testées sur un autre choix des même catégories d'individus).

\subsection{Typologie des ménages et des objets de consommation}

Langlois a mené tout au long de sa carrière des études sur la typologie des ménages et des biens de consommations qui lui ont permis de proposer de nouvelles typologies fondées statistiquement et multidimensionnelles à la différence de beaucoup de regroupements classiques. Outre que ces typologies lui ont permis de mieux analyser la dynamique des classes sociales et des structures de consommation, elles l'ont amené à constituer des données individuelles originales basées sur ces regroupements de ménages et de dépenses, par une pseudo-panélisation des enquêtes de budgets de familles canadiennes. La dialectique de l'objet d'étude, du modèle qui permet de l'observer et des données devient beaucoup plus complexe que le simple empirisme tirant d'observations brutes quelques lois statistiques dont la stabilité ne peut être démontrée, étant liées à des contextes particuliers. La constitution de données adéquates s'avère comme une des réponses possibles à l'extension des analyses statistiques sur un corps de données qui minimise les biais de sélection et étend considérablement (sur plusieurs dizaines d'années) l'horizon temporel de l'étude, réduisant ainsi les biais de contexte.

Les travaux de Langlois diffèrent donc profondément de l'approche pratiquée par Bourdieu : l'assise sur les traditions de Boudon et Tocqueville lui permet d'éviter de vouloir enfermer ses travaux dans les limites d'une théorie générale qui ne pourrait, comme il fut avéré par l'exemple de Bourdieu, qu'être un cadre trop général et invérifiable empiriquement. Une telle théorie générale s'est accompagnée, dans les travaux de Bourdieu, de la recherche des questions et même des données qui permettent de le conforter ${ }^{29}$. Par ailleurs, cette modestie scientifique dans l'ouvrage en la tradition sociologique s'accompagne d'un travail mené personnellement, d'un bout à l'autre du travail empirique - même lorsque la phase de constitution des données est particulièrement longue -, et non pas de l'émiettement de ce travail entre des collaborateurs dont on recolle a posteriori les résultats (avec le risque d'aboutir à un

29. On connait l'épisode d'un film consacré à Bourdieu où celui-ci demande à un collaborateur de rechercher les données qui permettrait de contester les conclusions d'un modèle économique. 
patchwork désuni, si tous travaillent dans le cadre étroit d'une théorie générale et d'hypothèses posées a priori).

\section{Quelques éléments de discussion sur la méthodologie empirique de la sociologie et de l'économie de la consommation :}

\subsection{Position des débats méthodologiques}

On observe dans le cours de Bourdieu une curieuse absence de discussion des règles de test des modèles économiques, qui constitue pourtant l'une des critiques qu'on pouvait attendre de lui. La distinction entre les diverses sciences sociales peut être opérée par les questions et domaines concernés par chacune ou par leurs méthodes, principalement le mode de preuve qu'elles appliquent. Il existe en effet de nombreux recoupements dans les sujets traités entre philosophie, histoire, sociologie et économie, par ces deux dernières par exemple, alors que leur mode de preuve est plus essentiel dans leurs différences de méthode que le degré de mathématisation de leur analyse. Dans ce sens, c'est une des erreurs de Bourdieu dans ce cours que de critiquer l'inutilité et le danger des mathématiques dans le raisonnement économique, danger qu'il attribue au fait de réduire une réalité sociale multidimensionnelle (dont les dimensions économiques, ontologiques, sociologiques s'interpénètrent) à des objets mathématiques «maquillées en catégories scientifiques». En fait, la formalisation mathématique n'est souvent connue des chercheurs en sciences sociales que par le formalisme analytique du $18^{\text {ième }}$ siècle, tel qu'il se retrouve dans la mécanique céleste classique et les techniques d'analyse mathématique enseignées dans le secondaire: son fondement sur les propriétés topologiques des espaces (définies par les voisinages entre les points) et leurs structures algébriques, dans une connexion originale liée au problème étudié (par exemple dans la modélisation en géométrie Riemannienne) sont négligées. On est alors loin de concepts bien définis (de manière indépendante) auxquels s'appliquent les opérations algébriques classiques. Les mathématiques appliquées à une question économique ou sociologique ne l'écrasent pas si elle ne sert qu'à dérouler de manière simple les logiques complexes à l'œuvre dans cette question.

On pourra discuter par exemple la distinction proposée par Schumpeter (1954, première partie) entre l'économie et la sociologique, qui attribue à la sociologie le rôle de décrire et expliquer les bases institutionnelles et sociales ${ }^{30}$ sur lesquelles porteront les applications de la science économique, par exemple la nature des contrats de propriété, alors que l'analyse économique quant à elle traite des questions relatives au comportement des agents et à la nature des effets qu'ils engendrent par ce comportement (la sociologie ou la psychologie économiques s'occuperont de savoir comment les agents en sont venus à adopter ces comportements). Les effets engendrés par les comportements individuels sont en effet bien souvent de nature sociologique, et sont également analysés par l'économie récente des interactions sociales ou

30. «Analyse générale (incluant de nombreuses disciplines de sciences sociales se chevauchant et mal coordonnées des phénomènes sociaux (société, classe, relations de groupe, commandement...) ». Péguy est plus direct dans sa critique des travaux des premiers sociologues français (De la situation faite à l'histoire et à la sociologie dans le monde moderne, 1905) : «La sociologie est une histoire prétendument réformée » (c'est-à-dire supposée scientifiquement fondée - Péguy est un grand défenseur de la méthode historique qu'il examine dans sa thèse inachevée, Véronique). 
des préférences (besoins) endogènes. Nous adoptons donc le point de vue d'une distinction des sciences sociales, non par leur objet d'étude, mais par leur méthode.

De la méthode hypothético-déductive, fondée sur des prémisses évidentes, intuitives (ou fournies par d'autres sciences) prônée par Adam Smith, l'analyse économique passa par la critique de cette méthode par John Stuart Mill et l'Ecole classique, selon lesquels la méthode hypothético-déductive ne peut fournir que des connaissances conjecturales du fait des multiples causes perturbatrices : seule la méthode expérimentale permettra des découvertes infaillibles. Les deux pôles de l'analyse économique sont déjà posés, analyse logique ou induction empirique par l'expérience (étendue à l'analyse statistique de données constituées préalablement à l'étude) ${ }^{31}$. L'école marginaliste (Cournot, 1838 ; Menger, Walras, Jevons, Edgeworth, Marshall, 1870-80) développe, par la mathématisation de l'analyse, la possibilité de constituer une Economie Pure («domestication de la flexibilité théorique par des contraintes formelles ») affranchie des conditions particulières de son application. La mesurabilité supposée de toutes les variables du modèle (d'où une tendance à l'utilité cardinale jusqu'à Vilfredo Pareto) en fait un cas limite (sorte d'expérience de pensée), indiquant un mécanisme assez puissant et suffisamment général pour être applicable à tous les phénomènes (i.e. non conditionnée par des circonstances particulières - supposant donc que ces circonstances, quand on applique le modèle, ne changent pas les liens logiques du modèle). Cette formalisation des mécanismes économiques ouvre la voie vers une estimation des coefficients des modèles algébriques et un test des hypothèses fondées sur leurs valeurs : en ce sens, l'écriture mathématique des modèles économiques a permis la réconciliation des deux méthodes, méthode hypothético-déductive et empiriste, comme on le voit clairement dans l'œuvre, à la fois théorique et appliquée, de Walras ${ }^{32}$.

L'analyse économique moderne, dite du main stream, héritera de cette histoire, par une séparation claire du modèle théorique (choix et mesure des variables; spécification des fonctions de comportement et des contraintes de choix) puis de la validation empirique de ses hypothèses ou conclusions ${ }^{33}$ (estimation des paramètres, test statistique des hypothèses ou des conclusions logiques du modèle) et ne sera plus guère contestée après $1945^{34}$, bien que

31. On trouvera une analyse détaillée de l'évolution de la méthodologie économique dans l'ouvrage de Claude Meidinger (1994).

32. Chez Walras, cette économie pure pose les jalons d'études d'économie appliquée, comme il apparait dans le débat entre Léon Walras et Henri Poincaré (correspondance de Walras éditée par Jaffé) : Poincaré souligne en particulier qu'il ne suffit pas de poser des hypothèses, il faut encore en rendre compte : les tester. On ne peut identifier le modèle abstrait à la réalité. Par ailleurs, la mesurabilité des variables peut s'entendre assez généralement (mesure ordinale et non cardinale, de l'utilité par exemple). Un autre débat intéressant concernant l'économie théorique opposa Gérard Debreu et Maurice Allais, le premier s'attachant à construire un modèle aussi général que possible (au sens mathématique: usage d'hypothèses de convexité par exemple plutôt qu'une spécification précise des fonctions de comportement) alors qu'Allais tenait à la possibilité de calculer la valeur des effets, non simplement d'obtenir les résultats les plus généraux d'existence et de stabilité.

33. La validation des conclusions plutôt que des hypothèses étant suggérée en particulier par Milton Friedman, l'objectif du modélisateur étant de reproduire la réalité observée, indépendamment du réalisme des hypothèses.

34. Rappelons que Keynes se refusait à l'utilisation de l'analyse statistique pour l'estimation et le test de la théorie, refus qu'on retrouve assez généralement dans l'école autrichienne (en particulier chez Mises, et Hayek, 1933, chapitre 1, pp. 26-40). Il aura à ce sujet un débat violent avec Haavelmo dans les années 30. Ce refus provient sans doute de sa conviction, depuis son Treatise on Probabilities, que les agents économiques choisissent toujours en situation d'incertitude absolue, non probabilisable. Les raisons de son refus de l'usage des mathématiques (sauf pour positionner dans le Treatise on Money ou la General Theory quelques équations comptables) sont plus mystérieuses : Schumpeter avance que cela est lié sans doute à sa méconnaissance de la littérature économique. 
demeurent sur ce sujet des débats récurrents entre empiristes (Leontief proposant de ne pas privilégier le raisonnement déductif, contredit par Haavelmo qui remarque que l'on choisit les faits que l'on décide de considérer) ${ }^{35}$.

On peut trouver dans la littérature économique récente quatre dépassements à ces contradictions : le premier peut être fondé sur l'analyse d'Hayek : la société construit l'équilibre social en même temps que le modèle qui l'exprime sous la forme d'existence d'un équilibre, et donc la réalité observée sera conforme à ce que prédit le modèle (une solution comparable à celle proposée par Bergson de l'évolution conjointe de l'esprit et de la matière dans L'Evolution Créatrice). Le deuxième dépassement peut s'opérer par la méthodologie du comme-si (as if) proposée par Friedman, la question étant le degré de stabilité de cette analogie entre le modèle et la réalité. La troisième se fonde sur la théorie des préférences révélées : l'observation permet de tester directement (sans modèle) des hypothèses théoriques, comme l'ont proposé Samuelson (1938) et Jean Ville (1946) ${ }^{36}$, approche généralisée par Arrow (1959). Le quatrième dépassement se fait par l'expérimentation. Cahuc-Zylberbeg (2016) considèrent (un peu vite) que la science économique est devenue expérimentale. C'est quand même partiellement le cas, sous la forme de trois types d'expériences, expériences naturelles, expériences contrôlées, expériences en laboratoire.

L'analyse économique a donc affermi sensiblement sa méthodologie, sans qu'une seule méthode soit actuellement adoptée par tous les économistes ni dans la discipline sociologique pourtant proche, sans doute par le fait (c'est une remarque importante de Schumpeter ${ }^{37}$ ) que les deux disciplines ont grandi séparément. On notera la distance de ces quatre dépassements alternatifs avec la méthode adoptée par Bourdieu dans son cours, où il opère systématiquement (plutôt que de les former en fonction de son analyse projetée) l'importation de concepts et de mécanismes économiques (investissement, consommation, équilibre...) dont il critique par ailleurs l'aspect partiel, peu réaliste et contradictoire, pour, in fine, faire la proposition peu fondée, théoriquement et empiriquement, d'une Théorie générale des champs sociaux (fumeuse et imprécise).

\subsection{Comparaison au mode de preuve dans les sciences sociales}

De l'histoire à la sociologie puis à l'analyse économique, le mode de preuve se fait plus systématique, afin de produire un test robuste d'une hypothèse limitée (i.e. qu'il a été possible de séparer de son contexte). Cette précision s'accompagne d'une distanciation à la réalité vécue

\footnotetext{
35. La critique toujours renaissante du manque de relation du modèle au réel (par les économistes « atterrés » par exemple en France actuellement) tient généralement au refus de l'hypothèse de rationalité individuelle et de la validité de l'hypothèse d'un environnement concurrentiel, qui sont les deux principales critiques de Bourdieu dans son cours.

36. Notons que les économistes anglo-saxons continuent à attribuer à Hendrick Houthakker (1956) la preuve du théorème fondamental d'intégrabilité due à Jean Ville, stipulant les conditions mathématiques pour que les fonctions de comportement d'un agent économique proviennent d'une optimisation.

37. «Il n'est pas assuré qu'une collaboration plus étroite puisse être féconde ». On notera le contre-exemple du séminaire commun de Becker et Richard Pozner à Chicago et les travaux communs de Simon Langlois avec des économistes, tel un récent article dans une revue économique (Boelaert, Gardes et aLanglois, 2017).
} 
par les acteurs, qui ne sont plus, dans l'analyse statistique des économétriciens, que des types d'individus, projection sur l'espace modélisé d'individus réels.

L'historien se contente en effet d'établir une synthèse de toutes les informations existantes ou accessibles, formulant une thèse (par exemple sur les motivations de Ravaillac), puis cherchant à récupérer d'autres documents que ceux qu'il a déjà exploités afin de valider cette hypothèse. Il ne doit pas seulement connaître les instruments et les méthodes (comme certains universitaires selon Péguy: «d'artificiels petits jeunes-gens maigres, maniaques de l'érudition »), mais le contenu de la réalité (probité dans l'examen des faits historiques afin de ne pas les réduire à un schéma conceptuel). Il lui faut à tout moment porter un jugement sur le poids d'un fait afin de le comprendre par rapport au réel directement vécu, dans lequel l'historien est inséré. Un bon historien doit rester en contact par sa vie personnelle avec la vie sociale qu'il cherche à décrire.

Le sociologue quant à lui, considérant que les comportements sont socialement déterminés (Langlois dirait plutôt, dans la tradition boudonnienne refusant cette caractérisation holiste : «socialement situés en contexte »), ne peut néanmoins s'abstraire d'une description complète de la société, dont il suppose les relations interpersonnelles complexes et pour la plupart non observables. N'ayant qu'une connaissance imparfaite (souvent non mesurable) des évènements, il n'aura que rarement à sa disposition des lois pour les expliquer, qui recouvrent complètement ces évènements. En les conceptualisant, il s'efforce d'atteindre le moteur social de ces comportements et par là le fonctionnement global d'une société, un peu comme les linguistes ont cru retrouver les origines communes des langues occidentales dans une racine indo-européenne inconnue (cette ambition de Georges Dumézil est actuellement discutée par les spécialistes $)^{38}$. Ces analyses diffèrent donc d'une simple description évènementielle qui observe et transmet, comme des historiens cherchant simplement une synthèse, une image résumée du réel sans chercher à en expliquer tous les ressorts sociaux et le fonctionnement de l'ensemble de la société. L'analyse sociologique ne peut donc se limiter à une série, même structurée, de monographies dont aucune ne saura dévoiler des ressorts cachés commun aux phénomènes étudiés séparément. Néanmoins, l'impossibilité de séparer un comportement individuel des interactions sociales et des normes et structures sociales, partiellement inconnues, qu'il subit, empêchera le plus souvent l'organisation de tests ponctuels d'une hypothèse clairement exprimée. Sa méthode passera donc généralement par des enquêtes complémentaires, suivies de l'analyse de ces enquêtes. Cette analyse a posteriori amène le social des sociologues à être un réel figé : or, « Le social est aussi du vital » (Bergson). Dans la réalité, il y a des lignes de faits dont aucune ne suffirait par elle-même à déterminer une vérité, mais qui pourrait la préciser par leur intersection - addition de probabilités. Il faut donc déterminer les raisons probables des comportements, et éventuellement calculer certains des paramètres qui les régulent au sein de lois partielles et conditionnelles (mais sans un modèle structurel comme celui que construiront les économistes du mainstream, ou du moins sans développement formel du modèle estimé à partir du modèle structurel).

38. On note le même effort chez les structuralistes : il existe des structures inconscientes de l'esprit, qu'il faut retrouver en construisant les matrices cachées des normes et des actions qui en dévoilent leurs structures profondes. 
Il est intéressant de comparer ces difficultés épistémologiques rencontrés par les sociologue à la méthode suivie par la philosophie réaliste, qu'on considérera dans l'œuvre de Bergson ${ }^{39}$. Bergson avance qu'il est possible de développer une intuition «créatrice » d'une réalité cachée, qui dépasse un simple enchaînement mécanique de causes indépendantes : il existe un ordre caché de ce qu'on cherche à comprendre et « si la vie est une création, on peut, par analogie, nous la représenter avec les créations que nous accomplissons nous-mêmes ». Un exemple de ce type d'analyse est donné dans sa Notice sur Ravaisson (Bergson in La Pensée et le Mouvant, 1934 ; (Euvres 1963 p.1471-2) : plus le travail de la nature est intense, plus l'œuvre produite est belle ; si la beauté nous livrait son secret, nous pénètrerions dans l'intimité du travail de la nature ; il y a donc une possibilité de remonter à cette source si l'on considère que la nature est elle-même un effet d'une cause : compréhension de la grâce et en conséquence de la beauté. «Toute chose manifeste, dans le mouvement que sa forme enregistre, la générosité infinie d'un principe qui se donne ».

La première tâche de ce type d'étude consiste à rejeter certaines évidences définitivement, puis à ne pas se laisser aller à déduire paresseusement des conséquences selon les règles d'une logique rectiligne (tangente à la courbe de la pensée). A chaque moment de la réflexion, il convient se défendre par une négation intuitive : «Devenu extérieur à lui-même, le penseur rentre en lui quand il revient à l'intuition ». C'est le même mouvement que celui de l'artiste saisissant l'aspiration fondamentale de la personne dans le portrait (Ravaisson, $L a$ Pensée et le Mouvant, p. 1504) : concentrer le portrait atour de la pensée latente et de l'âme régénératrice et non sur les contours matériels du modèle. Une analogie avec une étude économique est la considération de la force latente qui entraîne la dynamique économique (selon Marx par la domination liée à la possession du capital économique ; selon Adam Smith, par la concurrence : ce sont là des forces fondamentales qu'on cherchera à retrouver dans leurs manifestations - mais sans pouvoir les mesurer précisément).

A la différence des philosophes qui sautent d'une branche à l'autre de leur arbre dialectique, sans trop se soucier d'autre vertu que de leur agilité qui ne se nourrit pas d'une méditation continue, et en particulier de leur fidélité à la réalité, Bergson fait donc constamment référence à sa méthode («Si notre méthode est correcte», «La méthode qui nous parait d'ordinaire la plus sûre ») qui, comme il l'espère, lui permet de rester fidèle au réel. Dans l'analyse du fait religieux par exemple, il propose (Les Deux Sources de la Morale et de la Religion, 1932 ; Cuvres 1963 p. 182) comme première méthode envisageable de «considérer une certaine fonction de l'esprit qu'on peut observer directement, sans s'occuper de la répartition du réel en concepts correspondant à des mots» (ce sera le témoignage des mystiques) ; une seconde voie vise à embrasser tout ce que ce fait recouvre en termes de mots (prédécoupés dans la réalité), mais il faut alors «distinguer les illusions fondamentales » et s'attacher à « recouper deux lignes de faits » (p. 263) : en effet, « chacune ne fournit que la direction de la vérité parce qu'elle ne va pas assez loin : en prolongeant deux d'entre elles jusqu'au point où elles se coupent on arrivera pourtant à la vérité même ». La première méthode,

39. Ces considérations sont issues d'une lecture complète de l'œuvre publiée de Bergson, sans référence à des travaux d'autres auteurs discutant sa méthode : c'est donc une vue personnelle sans doute partielle. 
la plus sûre, s'accomplit par un effort de compréhension intérieur (introspectif) profond. En effet,

la philosophie (en ce sens inférieure aux mathématiques et aux sciences de la nature) doit partir de la désarticulation du réel qui a été opérée par le langage et qui est peut-être toute relative aux besoins de la cité : trop souvent elle oublie cette origine, et procède comme ferait le géographe qui, pour délimiter les diverses régions du globe et marquer les relations physiques qu'elles ont entre elles, s'en rapporterait aux frontières établies par les traités. (Bergson, 1963, p. ?)

\subsection{Sur divers problèmes de la preuve statistique en économie (et plus généralement pour toutes les sciences sociales)}

L'estimation et le test des modèles économiques, dont on peut dater les débuts systématiques à l'après-guerre, a rencontré une série de difficultés d'ordre technique qui, sous une forme apparemment purement statistique, correspondent tous à des problèmes méthodologiques rencontrés par toutes les sciences sociales. On notera qu'aucune de ces questions fondamentales n'est abordée dans le cours de Bourdieu, du fait de la quasi absence de discussion méthodologique organisée. Il n'est donc pas inutile de décrire succinctement certains de ces problèmes statistiques pour révéler en creux la limite des critiques formulées dans le cours à l'encontre de la discipline économique.

Les biais d'endogénéité, qui interdisent d'estimer les valeurs vraies des paramètres (et donc perturbent à la fois l'estimation des modèles et leur test) sont causés par une corrélation théorique (issue du modèle ou des erreurs de mesure des variables explicatives) de l'erreur statistique (produit combiné de toutes les causes latentes, c'est-à-dire non renseignées statistiquement) avec l'ensemble des variables explicatives - corrélation qui vient donc en négation directe de l'hypothèse d'orthogonalité entre ces deux termes fondant l'estimation par les moindres carrés ordinaires. Une telle endogénéité provient d'une causalité circulaire entre la variables expliquées et les variables explicatives, les variables latentes (liée aux « causes perturbatrices » de Stuart-Mill) composant le résidu étant corrélées aux variables explicatives du modèle. C'est donc la causalité circulaire entre les variables choisies pour modéliser le phénomène qui est ici en cause, problème classique de toutes les sciences sociales. Un tel biais a par exemple été repéré dans les analyses de consommation (Gardes et al., 2005) par la différence des estimations opérées sur enquête ou en séries temporelles. L'existence de variables latentes liées à certaines variables explicatives peut s'interpréter par l'existence d'un coût virtuel endogène attaché à la variable explicative en cause, par exemple pour les dépenses alimentaires à domicile (respectivement à l'extérieur) un prix virtuel du temps corrélé positivement (respectivement négativement) au revenu (mesuré en cross-section, c'est à dire statiquement le long de la distribution des revenus), mais dont la corrélation disparait en dynamique, si les individus comparés à deux périodes différentes ne diffèrent pas dans leur localisation sur la distribution du revenu (donc ont le même revenu relatif). Une procédure statistique décrite dans l'article permet de mesurer les changements de ce prix virtuel entre les ménages d'une même enquête, donc de fournir une explication statistique à l'endogénéité et un 
moyen de la corriger, en tenant compte de ces prix virtuels dans l'estimation ${ }^{40}$. On constate donc clairement dans cet exemple qu'un problème classique des sciences sociales, lié à l'impossibilité de contrôler toutes les causes annexes au phénomène étudié, trouve sa traduction statistique et des méthodes permettant de le prendre en compte, et éventuellement de révéler le poids de ces causes annexes par le biais de prix virtuels.

L'existence d'une dépendance au chemin (path dependency) interdit la statique comparative dans la mesure où deux observations atemporelles ne peuvent être comparées qu'en stipulant les cheminements alternatifs qui ont pu les transformer l'une en l'autre. C'est évidemment un problème essentiel pour la sociologie et l'économie qui utilisent assez généralement des enquêtes transversales pour tester leurs explications, les données de panel (avec une périodicité correspondant au cheminement réel des acteurs) étant rarement disponibles. Une modélisation des choix de consommation des ménages en géométrie Riemannienne (Gardes, 2021a) permet de traduire les différences de conditionnement des individus observés dans une enquête par un système de prix endogènes (des prix généralisés générant les décisions des agents dépendant de leur localisation sociale) dont l'existence peut être repérée par la courbure de l'espace Riemannien. L'examen des caractéristiques géométrique de cet espace permet de montrer l'existence d'une telle dépendance au chemin dans les évolutions de consommations liées aux changements de prix et de revenu. Une généralisation du modèle d'un espace euclidien (sans dépendance au chemin) à un espace Riemannien permet donc de tenir compte des conditionnements liés au cheminements différenciés des ménages et d'expliquer ainsi que les analyses statiques des consommateurs engendrent des interprétations biaisées de l'effet des différentes variables économiques et sociodémographiques.

Un troisième problème important intéresse toute étude opérée sur des données individuelles, qu'elle soit sociologique ou économique : il s'agit du biais de sous-estimation des variances des paramètres estimés (et donc sur tous les tests statistiques) en données nombreuses (de plusieurs milliers d'observations - le cas typique des ensembles de données microéconomiques actuellement utilisés). Les données nombreuses sont en effet caractérisées par des autocorrélations spatiales (concentration de l'information qu'on peut justifier par exemple par le théorème de Borsuk-Ulam, voir Gardes, 2021b) dont la méconnaissance implique une sousévaluation dans l'estimation des écart-types des coefficients estimés (fréquemment dans un rapport de 1.5 à 7). Tous les tests statistiques sont donc biaisés dans la comparaison des significativités dans divers échantillons dans la mesure où la significativité dépend de la taille (et de la structure plus ou moins concentrée) de l'échantillon. Ce problème intéresse donc toute analyse statistique menée sur des données individuelles, et tend à fournir systématiquement sur de telles données un résultat défavorable à tout test défini par l'égalité d'un paramètre à une valeur prédéterminée (l'expansion de l'échantillon permettant d'augmenter la probabilité de ce résultat négatif). Il suffit donc de formuler l'hypothèse dans ce sens («l'élasticité est positive et infra-unitaire ») pour la valider. Cette propriété statistique montre que la validation d'une

\footnotetext{
40. Les biais d'endogénéité reçoivent par ailleurs classiquement des solutions statistiques par l'instrumentation des variables explicatives endogènes, l'estimation par un système d'équations simultanées (la variable explicative endogène étant expliquée par une de ces équations) ou la disparition des causes annexes par une procédure expérimentale.
} 
hypothèse dépend de manière cruciale de la taille et de la structure de l'échantillon, qu'il faut donc considérer lorsqu'on compare les résultats de deux estimations.

L'analyse d'autres problèmes plus classiques communs aux analyses sociologiques et économiques, issus de la nature des données ou des choix méthodologiques de la significativité statistique, montrerait que la plupart des problèmes méthodologiques repérés dans les analyses économiques modernes se retrouvent en sociologie : les biais d'agrégation et l'analyse causale sur variables agrégées ${ }^{41}$, les biais de sélection liés à une sélection endogène de la population échantillonnée (Heckman, 1979, le choix de la limite statistique (discussion de la p-value à 5\% par McCloskey et Zyliak, 2009 qui signalent l'intérêt du niveau des paramètres, pas seulement de leur significativité (par exemple pour définir l'âge optimal de détection des cancers) par la définition d'une fonction de perte (loss function) qui dépendent de ces deux dimensions niveau de significativité statistique et importance des paramètres ou des écarts).

C'est donc, pensons-nous, sur le plan de la méthode statistique du test que les deux sciences peuvent se rejoindre afin qu'il soit possible de faire dialoguer leurs conclusions.

\subsection{Trois développements récents des analyses économiques de la consommation recoupant des problèmes sociologiques classiques}

La théorie économique de la consommation des ménages a récemment connu trois développements intéressants qui tous trois concernent des problèmes entendus auparavant comme de nature sociologique, où une analyse statistique sur des données plus riches (des panels ou pseudo-panels de consommateurs) permet des tests d'hypothèses robustes: l'hypothèse de rationalité est vérifiée par des tests non paramétriques dans le cadre des préférences révélées, ce qui permet de s'affranchir de spécifications particulières des fonctions de demande, et de considérer ainsi une rationalité beaucoup plus générale de l'agent, s'appliquant à tous les types de décision auxquels il est confronté ${ }^{42}$. Un autre ensemble de travaux (Gardes, 2019; voir également les deux modèles présentés en dernière section) a montré que la contrainte de temps intervient de manière essentielle dans les choix familiaux, tant de travail marchand ou domestique que dans l'usage des ressources financières. Ces travaux montrent que les résultats des études sociologiques sur les choix économiques des familles montrant que ces choix dépendaient de manière essentielle de la structure familiale (qu'une simple échelle de consommation ne permet pas de traduire adéquatement) recevaient une confirmation dans les études de l'allocation du temps initiés par Gary Becker. On connait enfin le développement des théories du modèle collectif (Browning et al., 2014) qui permettent de tenir compte des processus de décision complexes au sein des ménages.

\footnotetext{
41. Rappelons, principe que nous adoptons, que selon Hayek : seules les causes microéconomiques doivent être considérées : il n'y a pas de causalité pour des comportements repérés à un niveau macroéconomique.

42. Le résultat de deux études récentes est ainsi que les irrationalités détectées correspondent majoritairement à des individus soumis à de fortes perturbations connexes (par exemple une modification de la structure familiale) ou liées à des processus de choix collectifs non optimaux plutôt qu'à des irrationalités individuelles.
} 
On peut se demander enfin s'il est possible de construire un indicateur de vérisimilitude des modèles (permettant d'en comparer la pertinence), qui constituerait un mode de comparaison des explications sociologiques et économiques d'un même phénomène. L'erreur de la proposition logique que Popper propose dans La logique de la découverte scientifique (voir à ce propos Meidinger, 1994) comme la difficulté de construire un test pour des hypothèses non emboîtées, montrent que cette comparaison ne peut être qu'assez générale et qualitative. La construction d'expériences tenant compte des deux modélisations, sociologique et économique, permettrait cette comparaison, mais on ne peut déjà considérer actuellement que l'économie est désormais une science expérimentale. Ce n'est que partiellement exact bien qu'on puisse constater un développement considérable du retracement des causalités sur données individuelles, tenant compte des conditionnements (voir par exemple les travaux d'Heckman (2005) ou, dans l'ouvrage d'Angrist-Pischke (2009), l'exemple des analyses du marché du travail opérées sur des cohortes nées avant ou après l'applicabilité de la réforme du recrutement militaire ou celui du niveau des élèves jeunes ou vieux par rapport au reste de la

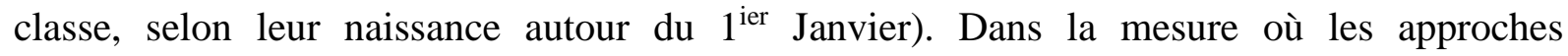
sociologiques et économiques restent à ce jour non intégrées et complémentaires, on peut, dans la tradition initiée par Gary Becker, non pas appliquer une théorie ou un modèle économique à une question sociologique ${ }^{43}$, mais un mode d'analyse lorsque le problème sociologique peut s'interpréter en terme de rareté (c'est d'ailleurs ce qu'a fait Bourdieu lui-même). Par exemple, l'inscription des choix individuels dans un espace où la localisation des agents détermine le système des prix et coûts généralisés auxquels leurs choix sont soumis, peut être décrit par des prix virtuels (traduisant les coûts issus de variables déterminantes cachées) et plus généralement par l'analyse dans une géométrie Riemannienne où les localisations (socio-économiques) des agents s'accompagne de conditions de choix (donc de coûts et de prix) différenciées (on trouvera cette analyse dans Gardes, 2021a). Il est également possible de proposer une définition de la distance sociale fondée sur le laps de temps qui serait nécessaire à un individu, dans les conditions où il se trouve (qui indiquent en particulier comment ses caractéristiques évoluent et quels sont les effets de ces évolutions sur les comportements étudiés), pour passer d'une localisation sociale à une autre (Gardes, 2021c). Le premier modèle indique en un sens comment traduire la différenciation sociale des conditions de choix des agents par des prix généralisés (nommés prix endogène pour indiquer qu'ils dépendent à la fois de la localisation sociale de l'agent et de ses choix antérieurs), permettant donc une analyse d'inspiration économique de ses choix; le second propose le calcul d'une distance sociale qui peut être considérée par la suite comme un déterminant des choix des agents ou de leurs interactions sociales.

\section{Conclusion}

\footnotetext{
${ }^{43}$ De même, les tentatives pour interpréter des phénomènes économiques à l'aide de concepts de la physique (en l'occurrence quantique pour plusieurs études récentes) ou biologiques donnent des résultats bien décevants et trompeurs.
} 
On peut regretter que le cours que Bourdieu a consacré à une critique de fond de la théorie économique et à la synthèse de l'ensemble de ses travaux concernant la consommation des ménages, ne fasse qu'un bilan très partiel de cette théorie. On note par exemple l'absence, dans les analyses critiques de la théorie économique présentées par Bourdieu dans ce cours, d'une prise en compte précise des évolutions de la discipline : émergence des concepts, émergence et différenciation des marchés, progrès du test statistique, analyse économique des préférences et contraintes sociales (économie du droit par exemple) ...

Par ailleurs, le débat entre la théorie pure et ses applications n'est guère approfondi. La théorie pure a été démontrée comme possible par l'économie mathématique et les analyses d'équilibre général, mais est-elle utile à la compréhension des marchés partiels et à la décision publique ? En particulier, le théorème de Debreu-Mantel-Sonnenshein, qui stipule que toute fonction particulière de comportement individuel peut se déduire d'une optimisation (et donc que le principe d'optimisation, c'est-à-dire d'intégrabilité des fonctions de comportement, ne permet pas de décrire la structure comportementale des modèles économiques), ne détruit-t-il pas l'intérêt d'une théorie fondée sur la rationalité des agents et leur pouvoir d'optimisation?

Enfin, le projet d'une science complète de la société oblige à des généralités et à l'utilisation de concepts flous et malléables qui s'adaptent mal aux applications empiriques détaillées. On observe une contradiction permanente entre l'aspect apparemment nouveau et complexe de ses concepts et l'aspect sommaire des applications qu'il en fit. C'est là une nécessité qui se pose à toutes les sciences sociales, limiter les ambitions de la théorie pour pouvoir assurer une procédure de test de l'explication théorique, qui ne peut concerner qu'une partie du schéma général, séparé par hypothèse des autres composantes du modèle supposées être orthogonales à l'hypothèse ou la conséquence qui est testée. Ceci montre qu'il est essentiel de définir précisément les modalités de mesure des concepts constituant l'espace sur lequel le modèle est développé, et les procédures de preuve qui seront appliquées à cette partie séparée du modèle ou de comparaison à d'autres modèles. L'ensemble des travaux de Langlois, nourris par l'œuvre de Boudon autant que par la méthodologie de la sociologie quantitative américaine, et bien assis sur le corpus théorique robuste de la sociologie moderne, montre qu'un projet unifié par la méthode et l'usage ordonné des techniques statistiques modernes (incluant la constitution de nouvelles données dédiées au problème étudié) fournit des résultats qu'on peut considérer comme plus durables et moins dépendants d'a priori théoriques dont on constate qu'ils apparaissent rapidement datés.

\section{Bibliographie}

Alexander, J., 1995, Fin de Siècle Social Theory : Relativism, Reduction, and the Problem of Reason, Verso ; traduction française : La Réduction. Critique de Bourdieu, Editions du Cerf, 2000.

Angrist, J.D., Pischke, J.S., 2009, Mostly Harmless Econometrics, Princeton University Press. Becker, G., 1962, Irrational Behavior and Economic Theory, Journal of Political Economy, vol. $70, n^{\circ} 1,1-13$. 
Becker, G., 1974, A Theory of Social Interactions, Journal of Political Economy, vol. 82, $\mathrm{n}^{\circ}$ 6, 1063-1093.

Becker, G., 1981, A Treatise obn the Family, Harvard University Press (enlarged edition 1991). Bergson, 1963, Euvres, édition du centenaire, Presses Universitaires de France.

Bollon, P., 2018, Bourdieu à Ciel Ouvert, Le Nouveau Magazine Littéraire, mars 2018.

Boelaert, J, Gardes, F., Langlois, S., 2017, Convergence des consommations entre classes socioéconomiques et contraintes non monétaires au Canada, L'Actualité économique, Revue d'analyse économique, vol. 93, no 4, décembre.

Bourdieu, P., Anthropologie Economique. Cours au Collège de France 1992-1993, Editions Raisons d'Agir-Seuil.

Bourdieu, P., 1979, La Distinction, Editions de Minuit.

Browning, M., Chiappori, P.A., Weiss, Y., 2014, Economics of the Family, Cambridge University Press.Cournot, , 1838, Recherche sur les Principes Mathématiques de la Théorie des Richesses, Hachette.

Cahuc, P., Zylberberg, A., 2016, Le négationnisme économique, Flammarion.

Gardes, F., 2021a, Endogenous Prices in a Riemannian Geometry Framework, Cahiers du Centre d'Economie de la Sorbonne.

Gardes, F, 2021b, Biases on Variances Estimated on Large Datasets, Cahiers du Centre d'Economie de la Sorbonne.

Gardes, F, 2021c, Synthetic time as a social distance, Cahiers du Centre d'Economie de la Sorbonne.

Gardes, F., 2019, The Estimation of Price Elasticities and the Value of Time in a Domestic Production Framework: an Application using French Micro-Data, Annals of Economics and Statistics, No. 135 (September 2019), 89-120.

F. Gardes, G.J. Duncan, P. Gaubert, C. Starzec, 2005, A Comparison of Consumption Laws Estimated on American and Polish Panel and Pseudo-Panel Data, Journal of Business and Economic Statistics, Vol. 23, No. 2 (Apr., 2005), pp. 242-253.

Gagné, G. et Langlois, S, 2002, Les Raisons Fortes: Nature et et Signification de l'Appui à la Souveraineté du Québec, Presses de l'Université de Montréal.

Gruel, L., 2005, Pierre Bourdieu Illusionniste, Presses Universitaires de Rennes.

Knight, F., 1919, Risk, Uncertainty and Profit, Hart, Schaffner and Marx Prize Essays.

Boston : Houghton Mifflin.

Joly, M., 2018, Pour Bourdieu, Editions du CNRS.

Heckman, J., 2005, The Scientific Model of Causality,Sociological Methodology, Vol. 35, pp. 1-97. Heckman, J., 1979, Sample Selection Bias as a Specification Error, Econometrica, vol. 47, n , January, 153-161.

Houthakker, H., 1950, Revealed Preference and the Utility Function, Economica, 17 (66), 159174.

Langlois, S., 1977, Les Réseaux Personnels et la Diffusion des Informations sur les Emplois, Recherches Sociographiques, vol. 18, $\mathrm{n}^{\circ}$ 2, 213-245.

Langlois, S., 2018, Evolution de l'Appui à l'Indépendance du Québec, in La Démocratie Référendaire dans les Ensembles Plurinationaux, sous la direction d'Amélie Binette et Patrick Taillon, Presses de 1'Université Laval, pp. 55-84.

Langlois, S., 2016, Le Québec Change, Del Busso Editeur.Deirdre McCloskey, D., Zyliak, S, 2008, The Cult of Statistical Significance, Princeton University Press. 
Magazine Littéraire, 1998, Pierre Bourdieu, l'Intellectuel Engagé, dossier spécial, n³69, Octobre : articles de Daniel Bensaïd, Didier Bezace, Pierre-Marc de Biasi, Patrice Bonnewitz, Dominique Colas, Philippe Corcuff, Daniel Bougnoux, Jean-Paul Dollé, François Dubet, Claude Grignon, Frédéric Martel, Philippe Meirieu, Olivier Mongin, Jean-Claude Monod, Janine Mossuz-Lavau, Philippe Raynaud, François de Singly.

Hayek, F.A., 1933, Monetary Theory and the Trade Cycle, édition en allemand 1929.Meidinger, C., 1994, Science Economique: Questions de Méthode, Editions Economica.

Mises, Ludwig von, 1963, Human Action, Yale University Press, revised edition.

Neary, J.P., Roberts, K.W.S., 1980, The Theory of Household Behaviour under Rationing, European Economic Review, 19, pp. 25-42.

Péguy, C., 1900, De la Grippe, Cahiers de la Quinzaine ; reproduit dans Les Euvres en Prose, Pléiade, tome 1.

Pribam, K., 1998, Les Fondements de l'Analyse Economique, Economica.

Samuelson, P.A., 1938, A Note on the Pure Theory of Consumer's Behaviour, Economica, 5 (17), 61-71.

Sartre, J.P., 1960, Questions de Méthode, in Critique de la Raison Dialectique, Gallimard.

Schumpeter, J.A., History of Economic Analysis, 1954.

Sciences Humaines, 2002, L'œuvre de Pierre Bourdieu: Sociologie, Bilan critique, Quel Héritage? numéro spécial.

Stigler, 1960, The Economics of Information, Journal of Political Economy, vol. 69, n³, 213 225.

Stigler, G.J., Becker, G.S., 1977, De Gustibus non est Disputandum, The American Economic Review, vol. 67, 2, 76-90.

Verdès-Leroux, J., 1998, Le Savant et le Politique, Essai sur le Terrorisme Politique de Pierre Bourdieu, Grasset.

Ville, J., 1946, Sur les Conditions d'Exitence d'une Ophélimité et d'un Indice du Niveau des Prix, Annales de l'Université de Lyon, 9, 32-39 ; traduit dans Review of Economic Studies, 19 (2), 123-128.

Ziliak, S., and McCloskey, D., 2009, The Cult of Statistical Significance: How the Standard Error Costs Us Jobs, Justice, and Lives, Ann Arbor, University of Michigan Press.

Travaux de Simon Langlois : voir en complément la bibliographie générale de l'ouvrage. 